\title{
Filigrane
}

Écoutes psychanalytiques

\section{Le travail clinique en contexte pédopsychiatrique : l'apport de la mentalisation à la pratique d'aujourd'hui}

\author{
Julie Achim et Miguel M. Terradas
}

Volume 24, numéro 2, automne 2015

Le devenir de la psychanalyse. Que nous disent les pratiques institutionnelles?

URI : https://id.erudit.org/iderudit/1036532ar

DOI : https://doi.org/10.7202/1036532ar

Aller au sommaire du numéro

\section{Éditeur(s)}

Revue Santé mentale au Québec

ISSN

1192-1412 (imprimé)

1911-4656 (numérique)

Découvrir la revue

Citer cet article

Achim, J. \& Terradas, M. M. (2015). Le travail clinique en contexte pédopsychiatrique : l'apport de la mentalisation à la pratique d'aujourd'hui. Filigrane, 24(2), 79-92. https://doi.org/10.7202/1036532ar

\section{Résumé de l'article}

L'évolution des situations cliniques ainsi que les changements institutionnels survenus au cours des dernières années au sein du réseau de la santé et des soins à l'enfance nous ont incités à revoir la nature de nos pratiques cliniques. Cet article présente une réflexion quant à la pertinence de la notion de mentalisation pour repenser les évaluations et interventions thérapeutiques offertes en contexte pédopsychiatrique. Ces nouvelles façons de faire visent à mieux tenir compte des difficultés et des ressources psychiques que présentent les enfants qui y consultent ainsi que leurs parents. Les constats cliniques ayant mené à cette réflexion sont d'abord exposés, la notion de mentalisation est ensuite définie et les principaux jalons de son développement normal et pathologique sont présentés. Les différentes modalités d'évaluation et d'intervention axées sur la mentalisation qui ont été développées et implantées au Service de pédopsychiatrie de l'Hôpital du Sacré-Coeur de Montréal sont présentées ainsi que le rationnel sous-jacent à ces modalités et les objectifs thérapeutiques visés. 


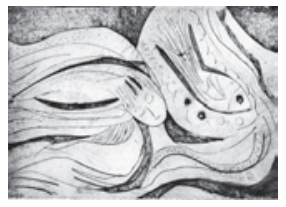

\title{
Le travail clinique en contexte pédopsychiatrique: I'apport de la mentalisation à la pratique d'aujourd'hui
}

\author{
Julie Achim et Miguel M. Terradas
}

\begin{abstract}
L'évolution des situations cliniques ainsi que les changements institutionnels survenus au cours des dernières années au sein du réseau de la santé et des soins à l'enfance nous ont incités à revoir la nature de nos pratiques cliniques. Cet article présente une réflexion quant à la pertinence de la notion de mentalisation pour repenser les évaluations et interventions thérapeutiques offertes en contexte pédopsychiatrique. Ces nouvelles façons de faire visent à mieux tenir compte des difficultés et des ressources psychiques que présentent les enfants qui y consultent ainsi que leurs parents. Les constats cliniques ayant mené à cette réflexion sont d'abord exposés, la notion de mentalisation est ensuite définie et les principaux jalons de son développement normal et pathologique sont présentés. Les différentes modalités d'évaluation et d'intervention axées sur la mentalisation qui ont été développées et implantées au Service de pédopsychiatrie de l'Hôpital du Sacré-Cœur de Montréal sont présentées ainsi que le rationnel sous-jacent à ces modalités et les objectifs thérapeutiques visés.
\end{abstract}

e texte présente les principales réflexions exposées lors d'une conférence ${ }^{1}$ prononcée dans le cadre du colloque Fondamentaux de la clinique institutionnelle: entre hier et demain? ayant eu lieu à l'Université de Caen (France) en avril 2014. Ce colloque réunissait de nombreux cliniciens issus de divers horizons afin de réfléchir aux profonds changements survenus au sein des institutions de soins au cours des dernières années ainsi qu'aux implications de tels bouleversements sur les pratiques cliniques. Nous avions répondu à l'invitation de Didier Drieu à nous joindre à ce groupe afin de partager nos constats quant à ces questions ainsi que nos réflexions quant à la pertinence de l'intégration de la notion de mentalisation à la pratique clinique en contexte pédopsychiatrique ${ }^{2}$. Le recours à cette notion, élaborée par Peter Fonagy et Mary Target, nous semble porteur d'avenues thérapeutiques prometteuses. En effet, il permet d'inscrire ces nouvelles façons 
de faire dans la continuité du travail d'allégeance psychodynamique réalisé auprès d'enfants et de leurs parents, tout en tenant compte des avancées scientifiques issues de la psychologie développementale, de l'observation du nourrisson et de la théorie de l'attachement.

Nous présenterons ici les différents constats cliniques de notre équipe ayant mené à cette réflexion, le rationnel sous-jacent aux aménagements thérapeutiques proposés, leur pertinence ainsi que certaines modalités d'évaluation et d'intervention axées sur la mentalisation privilégiées au Service de pédopsychiatrie de l'Hôpital du Sacré-Cœur de Montréal. Préalablement à la présentation de ces modalités, nous introduirons la notion de mentalisation que nous définirons brièvement. Nous présenterons ensuite son développement normal et pathologique afin d'être en mesure de bien situer les lieux de son achoppement, d'identifier des éléments cliniques en témoignant et de préciser les objectifs thérapeutiques qui en découlent.

\section{Constats cliniques et changements institutionnels à l'origine de cette réflexion}

Divers constats cliniques sont à l'origine de ces réflexions. Il y a d'abord la rencontre de plus en plus fréquente d'enfants présentant des problématiques multiples, complexes et souvent sévères, profitant peu des interventions thérapeutiques psychodynamiques classiques. Pensons notamment aux enfants présentant des tableaux cliniques atypiques, évoquant plusieurs catégories diagnostiques à la fois ou encore à ceux chez qui sont observées diverses pathologies externalisées du comportement. Mentionnons également les enfants qui présentent des traits de personnalité limite en émergence, des difficultés marquées à l'égard de la socialisation ou ayant subi de graves traumatismes relationnels. Malgré la diversité des difficultés ou des pathologies qu'ils présentent, nous constatons que ces enfants ont souvent en commun des défauts significatifs au plan de l'autorégulation et de la mentalisation. Par conséquent, nous observons chez eux un fonctionnement psychique et relationnel sous le signe de l'agir plutôt que de la parole, la pensée et la symbolisation.

Face à de telles difficultés, le recours aux outils thérapeutiques habituels semble peu adapté ou du moins, avoir une portée limitée puisque s'appuyant sur un équipement psychique que ces enfants, et bien souvent leurs parents, n’ont pas suffisamment développé. Cette situation confronte régulièrement les cliniciens œuvrant auprès d'eux à des impasses thérapeutiques, ce qui les laisse impuissants et à risque d'escalades au plan de l'utilisation 
des psychotropes qui peuvent devenir la seule avenue de traitement envisageable. Il importe donc d'inventer de nouvelles façons de faire et d'être qui tiennent compte de cette réalité clinique, et plus spécifiquement du niveau de développement psychique propre à ces enfants et à leurs parents. Nous sommes d'avis que ces nouvelles façons situent le travail thérapeutique en amont d'un travail psychanalytique plus classique et qu'elles impliquent l'adoption d'une posture différente par le clinicien.

La nécessité de revoir les pratiques apparaît d'autant plus prégnante que ces constats cliniques s'inscrivent dans un contexte de changements importants survenus au cours des deux dernières décennies au sein du réseau public québécois de la santé et des services sociaux offerts à l'enfance et à la famille. À la suite de la réorganisation des soins, le mandat confié à la pédopsychiatrie situe son apport au niveau de l'évaluation diagnostique différentielle en contexte de problématiques multiples, du traitement de patients nécessitant une prise en charge spécialisée et pluridisciplinaire ainsi qu’au niveau de la consultation auprès des partenaires du réseau.

C'est cette conjoncture qui nous a incités à repenser nos pratiques et à revoir les modalités de traitement à privilégier auprès de cette population. Dans ce contexte, les travaux de Fonagy et ses collègues consacrés au développement de la mentalisation chez l'enfant et à ses implications sur son fonctionnement psychique (pour une présentation détaillée de ces écrits, voir Allen, Fonagy, et Bateman, 2008) ainsi qu'à l'élaboration d'un traitement thérapeutique basé sur la mentalisation pour les adultes souffrant d'un trouble de personnalité limite (Bateman et Fonagy, 2004, 2006) sont apparus comme des contributions fort utiles, pouvant être importées et adaptées à la pratique pédopsychiatrique.

\section{La capacité de mentalisation : un élément essentiel pour se penser et penser l'autre}

La mentalisation désigne la capacité à percevoir et à interpréter son propre comportement et celui d'autrui en terme d'états mentaux (Allen et al., 2008). Ceci implique de concevoir que tout comme soi, les autres possèdent un monde interne constitué d'affects, besoins, désirs, pensées, intentions et motivations qui leur sont propres et qui sont à l'origine de leurs comportements. La mentalisation réfère ainsi à la capacité à considérer les états mentaux comme appartenant à la réalité interne donc subjective d'un individu plutôt qu'à une réalité objective et objectivable (Fonagy et Target, 1997). Elle implique également que l'individu conçoive que la subjectivité 
d'autrui lui échappe et qu'elle ne peut lui être pleinement accessible que si l'autre consent à lui partager ce qui l'habite. Ainsi, l'individu est conscient que la signification qu'il attribue aux conduites d'autrui demeure une hypothèse issue de sa propre expérience de l'autre et des indices contextuels (Fonagy, Bateman, et Luyten, 2012). Il est donc en mesure de considérer une situation à partir de différents points de vue et perspectives. Cette capacité lui confère une flexibilité lui permettant d'envisager que différentes motivations ou émotions puissent donner lieu à un même comportement.

Bien que l'enfant possède une certaine prédisposition innée à mentaliser, sa capacité à s'intéresser à ce qui l'habite et habite autrui se développe dans le cadre de la relation qu'il entretient avec sa principale figure d'attachement (Fonagy et al., 2012; Slade, 2005). Le processus par lequel elle se développe a longuement été décrit par Fonagy et Target à travers une série d'articles phares rédigés entre 1996 et 2007 — nous référons ici aux articles Playing with Reality I, II, III et IV. S'appuyant sur les idées de Winnicott, ils proposent un modèle du développement de la capacité de mentalisation. Ce modèle offre une compréhension du fonctionnement psychique de l'enfant de la naissance jusqu'à l'âge de cinq ans, allant au-delà d'une conception des enjeux propres à la relation mère-enfant pour s'intéresser plus spécifiquement aux modes de pensée qui prédominent chez l'enfant avant le déploiement de la capacité de mentalisation (Terradas et Achim, sous presse). Dans le premier, Playing with Reality I: Theory of mind and the normal development of the psychic reality (Fonagy et Target, 1996), les auteurs proposent un modèle du développement de la réalité psychique chez l'enfant. Ils évoquent l'existence de modes prémentalisants caractéristiques du fonctionnement mental du nourrisson puis du jeune enfant, soit les modes téléologique, équivalence psychique et fictif. Ces modes de pensée seraient associés à des façons distinctes de concevoir les liens existant entre les réalités interne et externe.

Le mode téléologique se développe entre la naissance et la moitié de la seconde année de vie. Il se caractérise par une considération simpliste et rationnelle des motifs sous-jacents à une action. Ce mode se fonde sur les observations que fait le jeune enfant à propos du comportement d'autrui. Ces informations lui permettent de faire ses premières inférences quant aux autres et à l'environnement, sans toutefois qu'il ne soit en mesure de prendre en compte leurs états mentaux. Sa compréhension de son propre comportement et de celui d'autrui se limite aux conséquences physiques - tangibles et observables - qu'il engendre; les motivations sous-jacentes à ces comportements ne sont pas considérées (Allen et al., 2008; Gergely, 2003). 
Le mode équivalence psychique se développe entre l'âge d'un an et demi et trois ans. Lorsque l'enfant fonctionne de façon prédominante sous ce mode, sa réalité interne prime sur la réalité externe. Ses états mentaux ne sont pas perçus comme des représentations de la réalité extérieure, mais bien comme un reflet exact de cette réalité. Réalité interne et réalité externe sont amalgamées. L'enfant demeure convaincu que ce qu'il pense et ressent correspond en tout point à la réalité externe. Il ne saisit pas encore que ce qui se déploie dans son esprit est le produit de l'interprétation que fait son esprit de la réalité externe (Allen et al., 2008; Gergely, 2003). Conséquemment, les pensées et les émotions, étant vécues comme réelles, peuvent devenir terrifiantes. Vers l'âge de trois ans, l'enfant développe une façon alternative de composer avec les réalités interne et externe, soit le mode fictif. Le développement de la capacité à faire semblant lui permet de comprendre que son monde interne diffère du monde externe. Cependant, tant que cette distinction n'est pas complètement acquise, ces deux univers se doivent d'être maintenus séparés puisque la confrontation à la réalité externe fait effraction et bloque le déploiement de l'imaginaire de l'enfant. En effet, le mode fictif existe en tant que réalité privée, complètement séparée de la réalité extérieure (Fonagy et Target, 2007) et éprouvée par l'enfant comme n'ayant aucune conséquence sur la réalité externe (Allen et al., 2008; Gergely, 2003). Bien qu' il soit capable de faire semblant, l'enfant n'est pas encore conscient qu'il le fait lorsqu'il joue (Gergely, 2003).

C'est l'intégration de ces modes de pensée prémentalisants qui témoigne de l'atteinte d'un mode de pensée réflexif ou mentalisant. Cette intégration se fait vers l'âge de quatre ou cinq ans. L'enfant comprend alors que les autres, tout comme lui, sont habités par des pensées et des sentiments - et donc par une réalité interne - qui sont reliés à la réalité externe, mais s'en distinguent. Target et Fonagy (1996) insistent sur l'importance du jeu de l'enfant comme contexte favorisant le développement de sa capacité de mentalisation. S'appuyant sur leurs observations cliniques, ils soutiennent que c'est notamment à travers le jeu que l'enfant saisit graduellement que ses états mentaux sont à la fois à l'image de sa réalité interne et de sa façon de se représenter le monde qui l'entoure. Ils insistent sur l'importance de la présence d'une figure d'attachement sécurisante qui, en contexte de jeu, peut identifier et retourner à l'enfant ses états mentaux sous une forme recevable. Cette présence soutiendra le développement de la capacité de l'enfant à établir le lien entre la réalité et ce qu'il met en scène à travers le jeu. En saisissant mieux son monde interne ainsi que le monde qui l'entoure, l'enfant 
arrivera à se comprendre lui-même et à comprendre autrui, à anticiper ses propres comportements et ceux des autres, à adapter ses réactions et, par conséquent, à s'autoréguler (Allen et al., 2008). Cette capacité lui confèrera également un sentiment de cohérence et de continuité de soi et d'autrui.

S'appuyant sur diverses notions élaborées par Winnicott et Bion, notamment la fonction miroir (Winnicott, 1971), la mère suffisamment bonne, le holding (Winnicott, 1963) ainsi que la capacité de rêverie maternelle (Bion, 1962), Fonagy et Target (1996) réferent fréquemment aux attitudes et comportements du parent contribuant à l'intégration des modes de pensée prémentalisants et donnant naissance au self réflexif. Lorsque le parent saisit ce qui habite l'enfant et y répond de manière sensible, il produit une réponse affective et comportementale à la fois semblable (puisqu'elle traduit la détresse de l'enfant) et distincte (puisqu'elle s'exprime de façon plus élaborée) de celle manifestée par l'enfant. Ainsi, pour que le reflet du parent permette à l'enfant de graduellement prendre conscience de ses états mentaux et de développer sa capacité de mentalisation, il doit être fait de manière suffisamment contingente, congruente, différenciée et marquée. La réponse du parent s'avère contingente lorsqu'elle est émise dans un délai suffisamment rapproché du comportement ou de l'état de l'enfant pour qu'il comprenne que les deux évènements sont liés. Le reflet du parent est congruent lorsqu'il cerne et traite de l'origine de la détresse de l'enfant. Il se différencie du comportement de ce dernier quand il est fondé sur l'état d'esprit de l'enfant et non sur celui du parent. Enfin, la réponse du parent est marquée lorsqu'elle est une version intentionnellement exagérée de la réponse émotionnelle normalement attendue qu'il donnerait face à l'état interne de l'enfant. Cette réponse permet à l'enfant de transformer son expérience interne brute, soit ses sensations physiques et ses réactions somatiques - ou représentation primaire - en une expérience représentée et interprétée par l'esprit — ou représentation secondaire (Fonagy, Gergely, Jurist, et Target, 2002). Il peut ainsi comprendre que son émotion est en fait une représentation modulable de l'état interne qu'il ressent. La mise en place de ce processus permet à l'enfant d'échapper à l'emprise unique de la réalité physique; il comprend alors que ce qu'il manifeste physiquement peut être représenté dans l'esprit de l'autre et avoir un sens. Ainsi, il apprend peu à peu à se représenter luimême ses états internes et réalise qu'il peut avoir une influence sur eux et sur ceux d'autrui.

Dans le contexte où l'enfant est victime de négligence ou d'abus, que la relation d'attachement parent-enfant est de type insécure ou encore lorsque 
le parent souffre de psychopathologie et présente d'importantes difficultés à s'intéresser au monde interne de l'enfant, le développement de la capacité de mentalisation de ce dernier sera compromise (Gergely, 2003). L'enfant ne pourra pas se construire une représentation de son univers psychique à partir de l'exploration de celui de son parent puisqu'il n'y aura pas accès. Il manquera de contenu disponible pour l'internalisation des états mentaux qui pourrait servir à l'élaboration d'une image cohérente du self. De plus, la maltraitance et l'abus peuvent causer une aversion à l'exploration du monde interne, les états mentaux du parent maltraitant ou abuseur pouvant devenir terrifiants (Allen, 2001). Par conséquent, l'enfant demeurera fixé à des stades prémentalisants empreints de distorsions cognitives compromettant sa compréhension de lui-même, d'autrui et de la relation qu'ils entretiennent (Allen et al., 2008). Ses capacités à penser et à se réguler demeureront fragiles et à risque de défaillance en contexte de stress (Allen, 2013).

Les résultats obtenus dans le cadre de quelques travaux empiriques récents appuient ce modèle théorique et suggèrent l'existence de liens entre une expérience d'abus et une faible capacité de mentalisation (Ensink, Normandin, Target, Fonagy et al., 2015; Terradas, 2007), ainsi qu'entre une faible capacité de mentalisation maternelle et la présence de symptômes psychopathologiques chez l'enfant (Ensink, Bégin, Normandin, et Fonagy, sous presse). C'est donc dire que les enfants évoluant dans un contexte peu favorable au développement de leur capacité de mentalisation seront à risque de présenter des difficultés au plan de la mentalisation et de l'autorégulation, mais également de développer des symptômes psychopathologiques.

Dans Playing with reality: III. The persistence of dual psychic reality in borderline patients, Fonagy et Target (2000) proposent une compréhension novatrice de la réalité psychique des patients dits limites en introduisant la notion de mentalisation. Les auteurs réfèrent aux individus qui rencontrent les critères diagnostiques d'un trouble de personnalité limite tel que conçu par les classifications catégorielles de type DSM (American Psychiatric Association, 2013), mais aussi à ceux qui sont à risque de régresser de façon marquée dans le contexte d'un traitement psychanalytique, de manifester des symptômes d'allure psychotique ou de provoquer d'intenses réactions contre-transférentielles chez le thérapeute. Les auteurs suggèrent que chez le patient dit limite, c'est le processus de métabolisation des angoisses de l'enfant par le parent qui aurait fait défaut. Selon eux, il est possible de croire que le parent, effrayé par les angoisses de l'enfant et incapable de les contenir, lui aurait reflété ses états mentaux sans modulation. Ce type de réponse 
traduit un fonctionnement prémentalisant sous le primat de l'équivalence psychique. Le parent aurait également pu éviter de refléter les angoisses de l'enfant, le laissant ainsi seul avec son expérience ou encore le faire de manière déconnectée, c'est-à-dire sans être en mesure de tenir compte de la nature des sentiments et des intentions qui l'habitent. Ce processus, qui évoque la dissociation dans le sens d'un manque de considération des éléments observables chez l'enfant ou pouvant être inférés à partir de son comportement témoignerait d'un fonctionnement caractéristique du mode fictif. La récurrence de ces reflets inadéquats compromettrait l'intégration de ces deux modes de pensée prémentalisants. Ainsi, les enfants ayant été mal mentalisés tendraient à démontrer une persistance des modes de pensée équivalence psychique et fictif qui alterneraient de manière brusque et soudaine et qui compromettraient leur capacité à penser leurs propres états mentaux et ceux d'autrui. Ces défaillances pourraient également engendrer le développement d'un alien self ou d'un noyau étranger au sein du self du patient limite qui serait aux prises avec des parties de lui inconnues, incongrues, voire redoutées.

\section{Interventions axées sur la mentalisation: une pratique clinique en plein essor, mais peu développée en contexte pédopsychiatrique}

Au cours des dernières années, plusieurs interventions ciblant différentes problématiques chez l'adulte ont intégré la notion de mentalisation. Le traitement basé sur la mentalisation pour les personnes souffrant d'un trouble de personnalité limite, élaboré par Bateman et Fonagy $(2004,2006)$ demeure le plus largement utilisé en contexte institutionnel. Cependant, à ce jour, rares sont les travaux concernant les applications de cette notion à la pratique clinique auprès des enfants et de leurs parents. À notre connaissance, seule une équipe hollandaise a proposé une intervention tenant compte de la mentalisation destinée aux enfants consultant en pédopsychiatrie (Verheugt-Pleiter, Zevalkink, et Schmeets, 2008). D'autres sont actuellement en cours de développement, notamment en Angleterre, en Suède et au Pays-Bas. Nos travaux se situent également dans cette lignée.

La pratique clinique auprès d'enfants consultant en pédopsychiatrie et auprès de leurs parents nous porte à croire qu'il y a chez bon nombre d'entre eux des défauts importants au plan de la capacité de mentalisation. En effet, nous constatons chez plusieurs d'entre eux diverses difficultés témoignant de tels déficits: fonctionnement sous le primat de l'agir et du ressenti 
corporel, difficultés au plan de l'autorégulation, difficultés à s'intéresser et à prendre en compte leur monde interne et celui d'autrui, difficultés à réfléchir voire à s'intéresser aux états mentaux sous-jacents à leurs propres comportements et à ceux d'autrui. Il en est également ainsi pour de nombreux parents qui ont du mal, même lorsqu'on les invite à le faire, à dépasser la simple description des comportements de leur enfant et à considérer ce qui motive ces comportements, à départager leurs propres conceptions et réactions de celles de leur enfant et, par conséquent, à considérer leur enfant comme un être pensant, habité par une vie intérieure qui lui est propre. Une première étude empirique exploratoire que nous avons menée auprès de parents dont les enfants consultent au Service de pédopsychiatrie appuie ces dernières observations cliniques et suggère que ces parents démontrent une capacité de mentalisation plus faible que celle attendue au sein de la population générale (Urfer, Achim, Terradas, Bisaillon, Dubois-Comtois et Lebel, 2015). Ces constats nous ont amenés à repenser nos évaluations et nos interventions afin d'être en mesure de mieux prendre en compte cette dimension importante du fonctionnement psychique chez l'enfant, mais également chez le parent.

\section{Interventions axées sur la mentalisation en contexte pédopsychiatrique : une illustration}

Nous travaillons actuellement à l'identification de manifestations cliniques témoignant d'une bonne capacité de mentalisation ou d'une capacité de mentalisation déficitaire chez les enfants et leurs parents. Diverses modalités d'évaluation s'avèrent pertinentes pour ce faire. Le contexte de l'évaluation clinique de l'enfant permet au thérapeute de porter une attention particulière à la manière dont l'enfant joue. Sa capacité et sa propension à mettre en scène une histoire issue de son imaginaire, d'en faire une narration incluant des états mentaux chez différents personnages ainsi que des liens entre ces états mentaux, les comportements des personnages et la nature de leurs interactions sont autant d'éléments témoignant de la capacité de mentalisation de l'enfant. Le Thematic Apperception Test ou T.A.T. ${ }^{3}$ (Murray, 1950) ainsi que les Histoires d'attachement à compléter ou HAC ${ }^{4}$ (Bretherton, Ridgeway, et Cassidy, 1990) nous semblent également des tâches propices au déploiement de la capacité de mentalisation de l'enfant. En effet, elles impliquent que le clinicien invite l'enfant à élaborer une histoire (et à la jouer dans le cas des HAC) à partir d'une image ou d'une amorce d'histoire impliquant une situation relationnelle affectivement chargée. L'enfant 
est implicitement (T.A.T.) ou explicitement (HAC) encouragé à raconter une histoire abordant ce qui pourrait habiter les personnages mis en scène et les motivations sous-tendant leurs comportements. La tâche impliquant que l'enfant doive tenir compte à la fois de l'objectivité du stimulus et de sa propre subjectivité, il est possible de croire que sa capacité de mentalisation sera mise à l'épreuve par cette consigne et que cette tâche permettra l'identification d'indices relatifs aux modes prémentalisants (p. ex., raconter une histoire sans tenir compte du percept pourrait représenter un indice de fonctionnement en mode équivalence psychique alors que décrire une histoire sans référence aux états mentaux pourrait être un indice de fonctionnement en mode fictif). L'identification d'indices cliniques témoignant de la prédominance de ces divers modes prémentalisants ou mentalisants, tel qu'élaboré par Domon-Archambault et Terradas (2015), s'avère fort utile afin d'être en mesure de cerner la nature des difficultés de mentalisation que présente l'enfant pour ensuite intervenir à ce niveau de façon plus spécifique et ainsi, soutenir la reprise du développement de la capacité de mentalisation. Mentionnons enfin l'évaluation relationnelle, une méthode développée au Service de pédopsychiatrie afin d'évaluer la qualité des liens parents-enfant. Inspirée des méthodes du Watch, Wait and Wonder ou 3W (Cohen, Muir, et Lojkasek, 2003) et du jeu trilogique de Lausanne ou LTP (Fivaz-Despeursinge et Corboz-Warnery, 1999), cette procédure implique d'inviter le parent à jouer avec l'enfant et à suivre le jeu initié par ce dernier. Ce contexte d'évaluation offre la possibilité au clinicien d'observer la capacité de l'enfant à jouer, mais aussi celle du parent à l'accompagner et le soutenir dans l'identification de ce qui habite les personnages mis en scène et ce qui pourrait motiver leurs comportements. Ceci permet le déploiement de ce que Target et Fonagy (1996) décrivent comme étant une condition cruciale au développement de la capacité de mentalisation de l'enfant, soit l'importance du jeu et de l'accompagnement du parent. Cette modalité s'inscrit dans un processus d'évaluation plus large de l'enfant. Ce dernier participe également à une activité de groupe à laquelle le parent assiste, via l'observation derrière un miroir sans tain, accompagné par la travailleuse sociale de l'équipe. Ce moment est l'occasion pour le parent de voir son enfant avec une perspective différente, décentrée de la sienne et de leur interaction, en étant accompagné par une clinicienne qui l'invite à s'interroger quant au sens que pourraient prendre les comportements de son enfant.

Diverses interventions thérapeutiques axées sur la mentalisation ont également été implantées. Dans le cadre de la psychothérapie individuelle, 
le jeu est utilisé comme outil permettant l'exploration des états mentaux des personnages mis en scène et l'élaboration de différentes hypothèses à cet égard. Pour ce faire, le thérapeute adopte une position plus active où il formule lui-même différentes hypothèses et cherche à amener l'enfant à s'intéresser à ce qui pourrait donner lieu à tel ou tel comportement lorsqu'il n'arrive pas à le faire lui-même (Terradas et Achim, 2013). Il en est de même pour certaines activités de groupe qui proposent le recours à différents médiums (p. ex., la narration d'un conte, la présence d'un animal) afin d'intéresser l'enfant aux états mentaux qui pourraient animer les différents protagonistes, à ce qui suscite de tels états mentaux et aux comportements auxquels ils peuvent donner lieu. Des interventions dyadiques ou familiales, dérivées respectivement des méthodes $3 \mathrm{~W}$ et LTP ainsi que du Reflective Family Play (Fivaz-Despeursinge, Philipp, Hayos, et Tissot, 2014) sont également privilégiées afin de soutenir le développement de la capacité des parents à penser leur enfant, à envisager ce qui pourrait l'habiter et conséquemment, à le lui refléter.

Mentionnons enfin l'adaptation (actuellement en cours) au contexte de pédopsychiatrie des interventions basées sur la mentalisation pour les éducateurs travaillant auprès d'enfants hébergés en Centre jeunesse développées par Domon-Archambault et Terradas (2015). S'appuyant sur le fait que l'éducateur est l'adulte qui accompagne l'enfant dans son quotidien, ils ont développé des interventions visant à la fois la promotion de la mentalisation à travers des activités éducatives ludiques ainsi que l'intégration des modes prémentalisants en un mode réflexif via des interventions cliniques ciblant spécifiquement ces différents modes de pensée.

Qu'elles se situent du côté de l'évaluation ou de l'intervention, ces diverses activités cliniques visent essentiellement à mieux cerner et à soutenir le développement de la capacité de mentalisation de l'enfant ainsi que celle du parent afin qu'ils soient en mesure de s'intéresser à leur monde interne et à celui d'autrui, à ce qui motive leurs réactions et comportements ainsi que ceux des autres. Ce travail implique l'adoption d'une posture différente d'une position psychanalytique classique. À l'instar de Bateman et Fonagy (2004, 2006) ainsi que d'Allen et ses collaborateurs (2008), nous pensons qu'il nécessite que le clinicien soit plus actif, qu'il explore spécifiquement les états mentaux du patient — ou ceux qui sont au cœur de son jeu — et qu'il propose des hypothèses quant à ce qui habite son patient (ou habite son jeu) lorsque ce dernier n'est pas en mesure de le faire seul. Nous croyons aussi qu'il implique que le clinicien utilise la relation thérapeutique autrement, 
soit pour penser les malentendus pouvant avoir lieu entre le patient et le thérapeute (Allen et al., 2008). En refaisant la séquence des évènements et des états mentaux qui en sont à l'origine, le thérapeute partage avec son patient ses perceptions et ses interrogations quant à ce qui a pu mener à une incompréhension. Ce faisant, il lui donne accès à son processus réflexif et permet ainsi au patient d'explorer les états mentaux d'une figure parentale bienveillante.

\section{Conclusion}

L'intégration de la notion de mentalisation à la pratique pédopsychiatrique nous semble des plus pertinentes pour tenter de se dégager des impasses thérapeutiques auxquelles nous confrontent régulièrement les enfants présentant des difficultés sévères ainsi que des profils diagnostiques multiples et complexes. Plusieurs raisons nous portent à penser qu'elle s'avère porteuse d'avenues prometteuses pour le travail en contexte pédopsychiatrique:

- parce qu'elle situe le travail thérapeutique à un niveau qui tient compte des difficultés marquées que présentent les enfants et leurs parents à penser leur expérience psychique;

- parce qu'elle vise à les intéresser à leur monde interne et à celui d'autrui;

- parce qu'elle permet également d'aider les parents à penser l'expérience psychique de leur enfant et se dégager de la seule considération des comportements observables;

- parce qu'elle permet au thérapeute de continuer à considérer l'enfant qui consulte dans sa globalité à une époque où les catégories diagnostiques s'avèrent les principaux repères pour comprendre les difficultés et orienter les interventions thérapeutiques.

$$
\begin{array}{r}
\text { Julie Achim } \\
\text { julie.achim@usherbrooke.ca }
\end{array}
$$

Miguel M. Terradas

\section{Notes}

1. Conférence prononcée par M. M. Terradas et J. Achim intitulée Enjeux de la clinique institutionnelle entre aujourd'hui et demain: l'apport de la mentalisation à la pratique clinique en contexte de pédopsychiatrie et de protection de la jeunesse.

2. Cette réflexion a été élaborée conjointement avec plusieurs collègues pédopsychiatres, psychologues, travailleurs sociaux du Service de pédopsychiatrie de l'Hôpital du 
Sacré-Cœur de Montréal ( $\mathrm{D}^{\mathrm{r}}$ Alain Lebel, Joanne Giasson, Isabelle Senécal, $\mathrm{D}^{\mathrm{r}}$ Sylvaine De Plaen, Émilie Deschenaux, Renée Hould et Alexandre Chabot) ainsi qu'avec notre collègue Karin Ensink, professeure à l'École de psychologie de l'Université Laval.

3. Pour une présentation détaillée de la pertinence du T.A.T. pour l'évaluation de la capacité de mentalisation, voir Haméon Denis (2015).

4. Pour une présentation détaillée de la pertinence des HAC pour l'évaluation de la capacité de mentalisation, voir Chabot, Achim, et Terradas (2015).

\section{Références}

Allen, J. G. (2013). Mentalizing in the Development and Treatment of Attachment Trauma. London: Karnac Books.

Allen, J. G. (2001). Traumatic Relationships and Serious Mental Disorders. Chichester: John Wiley et Sons.

Allen, J. G., Fonagy, P. et Bateman, A. W. (2008). Mentalizing in Clinical Practice. Washington, DC: American Psychiatric Publishing, Inc.

American Psychiatric Association (2003). Diagnostic and Statistical Manual of Mental Disorders (DSM 5), Fifth Edition. Arlington: American Psychiatric Publishing.

Bateman, A. W. et Fonagy, P. (2004). Psychotherapy for Borderline Personality Disorder: Mentalization-Based Treatment. Oxford: Oxford University Press.

Bateman, A. W. et Fonagy, P. (2006). Mentalization-Based Treatment for Borderline Personality Disorder: A Practical Guide. Oxford: Oxford University Press

Bion, W. R. (1962). A theory of thinking. International Journal of Psycho-Analysis, 43, 306-310.

Bretherton, I., Ridgeway, D. et Cassidy, J. (1990). Assessing internal working models of the attachment relationship. Dans M. T. Greenberg, D. Cicchetti et E. M. Cummings (dir.), Attachment in the Preschool Years (pp. 273-308). Chicago: The University of Chicago Press.

Chabot, A., Achim, J. et Terradas, M. M. (2015). La capacité de mentalisation de l'enfant à travers le jeu et les histoires d'attachement à compléter: perspectives théorique et clinique. La Psychiatrie de l'enfant, 58 (1), 207-240.

Cohen, N. J., Muir, E. et Lojkasek, M. (2003). The first couple: Using Watch, Wait and Wonder to change troubled infant-mother relationships. Dans S. M. Johnson et V. E. Whiffen (dir.), Attachment Processess in Couple and Family Therapy (p. 215-233). New York: Guilford Press.

Domon-Archambault, V. et Terradas, M. M. (2015). Les interventions fondées sur la notion de mentalisation auprès des enfants en situation d'hébergement. Revue québécoise de psychologie, 36 (2), 1-34.

Ensink, K., Bégin, M., Normandin, L. et Fonagy, P. (sous presse). Maternal and child reflective functioning in the context of trauma: Pathways to depression and externalizing difficulties. European Journal of Psychotraumatology.

Ensink, K., Normandin, L., Target, M., Fonagy, P., Sabourin, S., et Berthelot, N. (2015). Mentalization in children and mothers in the context of trauma: An initial study of the validity of the Child Reflective Functioning Scale. British Journal of Developmental Psychology, 33 (2), 203-217.

Fivaz-Despeursinge, E. et Corboz-Warnery, A. (1999). The Primary Triangle: A Developmental Systems View of Mothers, Fathers, and Infants. New York: Basic Books.

Fivaz-Despeursinge, E., Philipp, D., Hayos, C. et Tissot, H. (2014). Reflective Family Play: A brief family therapy combining the LTP with Watch, Wait, and Wonder. Dans E. FivazDespeursinge et D. Philipp (dir.), The Baby and the Couple: Understanding and Treating Young Families. Hove: Routledge. 
Fonagy, P., Bateman, A. W. et Luyten, P. (2012). Introduction and overview. Dans A. W. Bateman et P. Fonagy (dir.), Handbook of Mentalizing in Mental Health Practice (pp. 3-42). Washington, DC: American Psychiatric Publishing, Inc.

Fonagy, P., Gergely, G., Jurist, E. et Target, M. (2002). The social biofeedback theory of affectmirroring: The development of emotional self-awareness and self-control in infancy. Dans P. Fonagy, G. Gergely, E. Jurist et M. Target (dir.), Affect Regulation, Mentalization, and the Development of the Self (pp. 145-202). New York: Other Press.

Fonagy, P. et Target, M. (1996). Playing with reality: I. Theory of mind and the normal development of psychic reality. The International Journal of Psycho-Analysis, 77 (2), 217-233.

Fonagy, P. et Target, M. (1997). Attachment and reflective function: Their role in self-organization. Development and Psychopathology, 9 (4), 679-700.

Fonagy, P. et Target, M. (2000). Playing with reality: III. The persistence of dual psychic reality in borderline patients. International Journal of Psychoanalysis, 81 (5), 853-873.

Fonagy, P. et Target, M. (2007). Playing with reality: IV. A theory of external reality rooted in intersubjectivity. International Journal of Psychoanalysis, 88 (4), 917-937.

Gergely, G. (2003). The development of teleological versus mentalizing observational learning strategies in infancy. Bulletin of the Menninger Clinic, 67, 113-131.

Haméon Denis, F. (2015). Capacité de mentalisation d'enfants de 8 à 10 ans: proposition d'une grille d'évaluation convenant au T.A.T. Thèse de doctorat inédite. Université de Sherbrooke, Sherbrooke, Canada.

Murray, H. A. (1950). Manuel du Thematic Apperception Test. Paris: Centre de psychologie appliquée.

Slade, A. (2005). Parental reflective functioning: An introduction. Attachment \& Human Development, 7 (3), 269-281.

Target, M. et Fonagy, P. (1996). Playing with reality: II. The development of psychic reality from a theoretical perspective. The International Journal of Psycho-Analysis, 77 (3), 459-479.

Terradas, M. M. (2007). La fonction réflexive chez les mères d'enfants victimes d'abus sexuel: Développement d'une échelle mesurant le fonctionnement réflexif de la mère dans un contexte d'interaction mère-enfant. Thèse de doctorat inédite. Université Laval, Québec, Canada.

Terradas, M. M., et Achim, J. (2013). "Arrête de faire semblant, on joue pour vrai!» Intégration de techniques d'intervention basées sur la mentalisation à la psychothérapie d'enfants d'orientation psychanalytique. La Psychiatrie de l'enfant, 56 (2), 439-465.

Terradas, M. M. et Achim, J. (sous presse). Souffrances dans les liens parent-enfant: pratiques éducatives fondées sur la mentalisation en protection de l'enfance. Estilos da Clinica.

Urfer, F.-M., Achim, J., Terradas, M. M., Bisaillon, C., Dubois-Comtois, K. et Lebel, A. (2015). Fonction réflexive de parents d'enfants d'âge scolaire consultant en pédopsychiatrie: une étude pilote. Devenir, 28 (3), 149-178.

Verheugt-Pleiter, A. J. E., Zevalkink, J. et Schmeets, M. G. J. (2008). Mentalizing in Child Therapy: Guidelines for Clinical Practitioners. London: Karnac

Winnicott, D. W. (1963). The theory of parent and infant relationships. Dans P. Buckley (dir.), Essential Papers on Object Relations (pp. 71-101). New York: University Press.

Winnicott, D. W. (1971). Jeu et réalité. Paris: Gallimard. 